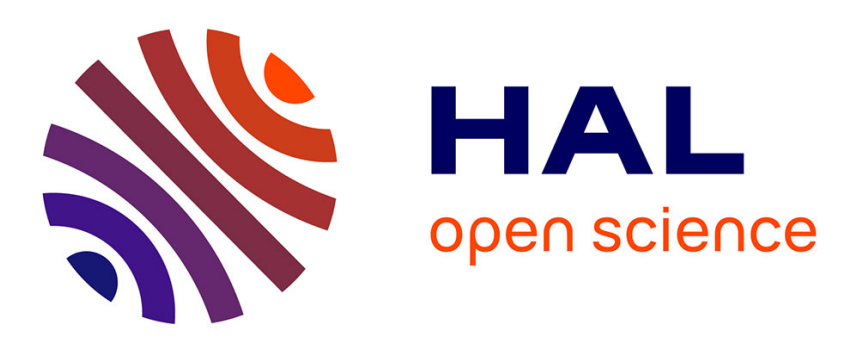

\title{
Kinetic evidence of magnetic reconnection due to Kelvin-Helmholtz waves
}

W. Li, M. André, Yu. V. Khotyaintsev, A. Vaivads, D. B. Graham, S. Toledo-Redondo, Pierre Henri, C. Norgren, Chen Wang, B. B. Tang, et al.

\section{- To cite this version:}

W. Li, M. André, Yu. V. Khotyaintsev, A. Vaivads, D. B. Graham, et al.. Kinetic evidence of magnetic reconnection due to Kelvin-Helmholtz waves. Geophysical Research Letters, 2016, 43 (11), pp.5635-5643. 10.1002/2016GL069192 . insu-01352463

\section{HAL Id: insu-01352463 \\ https://hal-insu.archives-ouvertes.fr/insu-01352463}

Submitted on 24 Apr 2017

HAL is a multi-disciplinary open access archive for the deposit and dissemination of scientific research documents, whether they are published or not. The documents may come from teaching and research institutions in France or abroad, or from public or private research centers.
L'archive ouverte pluridisciplinaire HAL, est destinée au dépôt et à la diffusion de documents scientifiques de niveau recherche, publiés ou non, émanant des établissements d'enseignement et de recherche français ou étrangers, des laboratoires publics ou privés. 


\section{Geophysical Research Letters}

\section{RESEARCH LETTER \\ 10.1002/2016GL069192}

\section{Special Section: \\ First results from NASA's Magnetospheric Multiscale (MMS) Mission}

\section{Key Points:}

- Magnetosheath electrons enter magnetosphere and magnetospheric electrons escape due to reconnection - Field-aligned heating of magnetosheath electrons at the high-density edges of the jets - Gradual ion mixing across the magnetopause

Correspondence to:

W. Li,

wyli@irfu.se

\section{Citation:}

Li, W., et al. (2016), Kinetic evidence of magnetic reconnection due to Kelvin-Helmholtz waves,

Geophys. Res. Lett., 43, 5635-5643, doi:10.1002/2016GL069192.

Received 15 APR 2016 Accepted 18 MAY 2016 Accepted article online 25 MAY 2016 Published online 11 JUN 2016

\section{Kinetic evidence of magnetic reconnection due to Kelvin-Helmholtz waves}

\author{
W. Li ${ }^{1}$, M. André ${ }^{1}$, Yu. V. Khotyaintsev ${ }^{1}$, A. Vaivads ${ }^{1}$, D. B. Graham ${ }^{1}$, S. Toledo-Redondo ${ }^{2}$, \\ C. Norgren ${ }^{1,3}$, P. Henri' ${ }^{4}$, C. Wang $^{5}$, B. B. Tang ${ }^{5}$, B. Lavraud ${ }^{6,7}$, Y. Vernisse ${ }^{6}$, D. L. Turner ${ }^{8}$, \\ J. Burch ${ }^{9}$, R. Torbert $^{10}$, W. Magnes ${ }^{11}$, C. T. Russell ${ }^{12}$, J. B. Blake ${ }^{8}$, B. Mauk ${ }^{13}$, B. Giles ${ }^{14}$, \\ C. Pollock ${ }^{15}$, J. Fennell ${ }^{8}$, A. Jaynes ${ }^{16}$, L. A. Avanov ${ }^{14}$, J. C. Dorelli ${ }^{14}$, \\ D. J. Gershman ${ }^{14,17}$, W. R. Paterson ${ }^{14}$, Y. Saito ${ }^{18}$, and R. J. Strangeway ${ }^{12}$
}

${ }^{1}$ Swedish Institute of Space Physics, Uppsala, Sweden, ${ }^{2}$ Science directorate, European Space Agency, ESAC, Madrid, Spain, ${ }^{3}$ Department of Physics and Astronomy, Uppsala University, Uppsala, Sweden, ${ }^{4}$ LPC2E, CNRS, Orléans, France, ${ }^{5}$ National Space Science Center, Beijing, China, ${ }^{6}$ Institut de Recherche en Astrophysique et Planétologie, Université de Toulouse (UPS), Toulouse, France, ${ }^{7}$ Centre National de la Recherche Scientifique, UMR 5277, Toulouse, France, ${ }^{8}$ Space Sciences Department, The Aerospace Corporation, El Segundo, California, USA, ${ }^{9}$ Southwest Research Institute, San Antonio, Texas, USA, ${ }^{10}$ Space Science Center, University of New Hampshire, Durham, New Hampshire, USA, ${ }^{11}$ Space Research Institute, Austrian Academy of Science, Graz, Austria, ${ }^{12}$ Department of Earth and Space Sciences, University of California, Los Angeles, California, USA, ${ }^{13}$ The Johns Hopkins University Applied Physics Laboratory, Laurel, Maryland, USA, ${ }^{14}$ NASA Goddard Space Flight Center, Greenbelt, Maryland, USA, ${ }^{15}$ Denali Scientific, Healy, Alaska, USA, ${ }^{16}$ Laboratory for Atmospheric and Space Physics, University of Colorado Boulder, Boulder, Colorado, USA, ${ }^{17}$ Department of Astronomy, University of Maryland, College Park, Maryland, USA, ${ }^{18}$ Japan Aerospace Exploration Agency, Tokyo, Japan

Abstract The Kelvin-Helmholtz $(\mathrm{KH})$ instability at the Earth's magnetopause is predominantly excited during northward interplanetary magnetic field (IMF). Magnetic reconnection due to $\mathrm{KH}$ waves has been suggested as one of the mechanisms to transfer solar wind plasma into the magnetosphere. We investigate $\mathrm{KH}$ waves observed at the magnetopause by the Magnetospheric Multiscale (MMS) mission; in particular, we study the trailing edges of $\mathrm{KH}$ waves with Alfvénic ion jets. We observe gradual mixing of magnetospheric and magnetosheath ions at the boundary layer. The magnetospheric electrons with energy up to $80 \mathrm{keV}$ are observed on the magnetosheath side of the jets, which indicates that they escape into the magnetosheath through reconnected magnetic field lines. At the same time, the low-energy (below $100 \mathrm{eV}$ ) magnetosheath electrons enter the magnetosphere and are heated in the field-aligned direction at the high-density edge of the jets. Our observations provide unambiguous kinetic evidence for ongoing reconnection due to $\mathrm{KH}$ waves.

\section{Introduction}

The magnetic reconnection process was long believed to be more significant and provide stronger solar wind-magnetosphere coupling during southward than northward interplanetary magnetic field (IMF) [Perreault and Akasofu, 1978]. However, substantial evidence was reported that more solar wind particles enter the magnetopause during northward IMF [Wing et al., 2014]. These solar wind particles generally lead to a thicker low-latitude boundary layer (LLBL), characterized by tailward moving plasma with intermediate properties between the magnetosheath and the plasma sheet [Mitchell et al., 1987], and a cold and dense plasma sheet (CDPS) [Terasawa et al., 1997], compared to a more common hot and tenuous plasma sheet. Possible mechanisms to transfer solar wind plasma from open magnetic field lines into closed geomagnetic field lines include high-latitude reconnection [Song and Russell, 1992], Kelvin-Helmholtz instability [Nykyri and Otto, 2001; Nakamura et al., 2008], and kinetic Alfvén waves [Chaston et al., 2007].

The Kelvin-Helmholtz instability can be excited at the boundary of two plasma regions if the velocity shear across the interface is large enough [Chandrasekhar, 1961; Miura and Pritchett, 1982], such as the low-latitude magnetopause situated between the magnetosheath, characterized by an antisunward flow of shocked solar wind, and the nearly stagnant magnetosphere. The excitation and evolution of the $\mathrm{KH}$ instability along the magnetopause boundary have been studied extensively with in situ observations [Sckopke et al., 1981; Hasegawa et al., 2004], localized simulation models [Ma et al., 2014; Nakamura and Daughton, 2014],
○2016. American Geophysical Union. All Rights Reserved. 
and global simulation models with a magnetosphere-ionosphere coupling system [Li et al., 2012, 2013]. The Kelvin-Helmholtz waves or vortices tend to be formed more frequently during northward than southward IMF along the low-latitude magnetopause [Hasegawa et al., 2006; Lin et al., 2014] and have been suggested to play an important role in the formation of the LLBL and CDPS [Hasegawa et al., 2004; Taylor et al., 2008].

The role of $\mathrm{KH}$ waves to generate LLBL and CDPS requires efficient entry of collisionless solar wind plasma into the magnetosphere; thus, there must be a process invoked by $\mathrm{KH}$ waves to break the frozen-in condition of ideal magnetohydrodynamics (MHD). MHD and particle-in-cell (PIC) simulations under various boundary conditions suggested two types of magnetic reconnection driven by $\mathrm{KH}$ waves. Type I reconnection occurs when the magnetic field components along the wave vector of $\mathrm{KH}$ waves are antiparallel across the velocity shear layer (antiparallel model) [Pu et al., 1990; Knoll and Chacón, 2002; Nakamura et al., 2008]. KH waves can increase the magnetic shear even if the boundary starts from a small magnetic shear angle and compress the current sheet down to the electron inertial scale [Nakamura et al., 2013]. Type I reconnection is commonly excited at the trailing or sunward edge of the $\mathrm{KH}$ wave and leads to direct plasma mixing across the shear layer. Here a trailing edge is represented by a crossing from the magnetosphere to the magnetosheath, and a reversed crossing indicates a leading edge. Three-dimensional (3-D) PIC simulations show that flux ropes can be generated by type I reconnection at the boundary, and their size and magnetic field structure are in agreement with THEMIS (Time History of Events and Macroscale Interactions) observations at Earth's low-latitude magnetopause [Nakamura et al., 2013].

Type II reconnection is driven at the leading or tailward edge when the velocity shear is sufficiently strong to generate highly rolled-up $\mathrm{KH}$ vortices, for parallel magnetic field conditions across the boundary (parallel model) [Nykyri and Otto, 2001; Nakamura and Fujimoto, 2006; Karimabadi et al., 2013]. Type II reconnection forms magnetic islands in 2-D simulation models both at the shear velocity boundary [Faganello et al., 2008] and inside KH vortices [Henri et al., 2012], and plasma can be transferred across the shear layer. The simulation study by Nakamura et al. [2008] indicates that both type I and type II reconnection may play a substantial role in plasma transfer and mixing across Earth's magnetopause, while linear theory analysis shows that type I reconnection is more likely to be generated during the $\mathrm{KH}$ wave evolution.

In a Cluster case study by Hasegawa et al. [2009], a current sheet of thickness a few times the ion-inertial length was found at a trailing edge of the $\mathrm{KH}$ wave, with a magnetic shear of $\sim 60^{\circ}$. Direct signatures of local reconnection were identified in the current sheet. The most remarkable evidence is an Alfvénic outflow jet parallel to the magnetosheath flow (positive jet) from both the ion instrument and the perpendicular drift velocity from electric and magnetic field measurements. Other signatures include a nonzero magnetic field component normal to the sheet and a field-aligned beam of accelerated electrons. However, a several second resolution for particle data (e.g., $3 \mathrm{~s}$ for THEMIS and $4 \mathrm{~s}$ for Cluster) was not sufficient to resolve detailed kinetic processes in the current sheet. At this point, there is no direct evidence of $\mathrm{KH}$ waves-induced reconnection based on particle data.

Unlike the reconnection process near the subsolar magnetopause, $\mathrm{KH}$ waves and possible reconnection processes propagate tailward at a speed about $200 \mathrm{~km} / \mathrm{s}$ in the spacecraft rest frame, implying the time duration of a cross of an ion scale reconnection region is $\sim 1 \mathrm{~s}$ or shorter. The high-resolution Fast Plasma Investigation (FPI) onboard the MMS mission [Burch et al., 2015; Pollock et al., 2016; Fuselier et al., 2014; Burch et al., 2016] provides the first opportunity to directly investigate particle evidence of reconnection due to $\mathrm{KH}$ waves. We study a long-duration Kelvin-Helmholtz wave event observed by MMS on 8 September 2015. This event is associated with reconnection jets and asymmetric magnetic and electric Hall fields [Eriksson et al., 2016]. Here we focus on the kinetic evidence of reconnection due to KH waves [Gosling et al., 1990].

\section{Overview of Kelvin-Helmholtz Waves}

An overview is presented in Figure 1 which shows the identified Kelvin-Helmholtz waves along the duskside magnetopause on 8 September 2015. Figures $1 \mathrm{a}-1 \mathrm{~d}$ show a prolonged steady solar wind plasma and IMF conditions in GSE (Geocentric Solar Ecliptic) coordinates from 09:15 to 11:40 UT, which is shifted to the bow shock, obtained from the OMNI data product at $1 \mathrm{~min}$ resolution. The solar wind velocity and the IMF direction are steady during the whole interval, while the dynamic pressure fluctuates slightly due to solar wind density variations. The IMF is northward with a nonzero $B_{X}$ component. The averaged solar wind parameters are: $10.0 \mathrm{~cm}^{-3}$ for number density, $[-509,-4,-11] \mathrm{km} / \mathrm{s}$ (GSE) for ion velocity, $5.18 \mathrm{nPa}$ for dynamic pressure, and $[12.7,2.9,16.3] \mathrm{nT}(\mathrm{GSE})$ for IMF. 

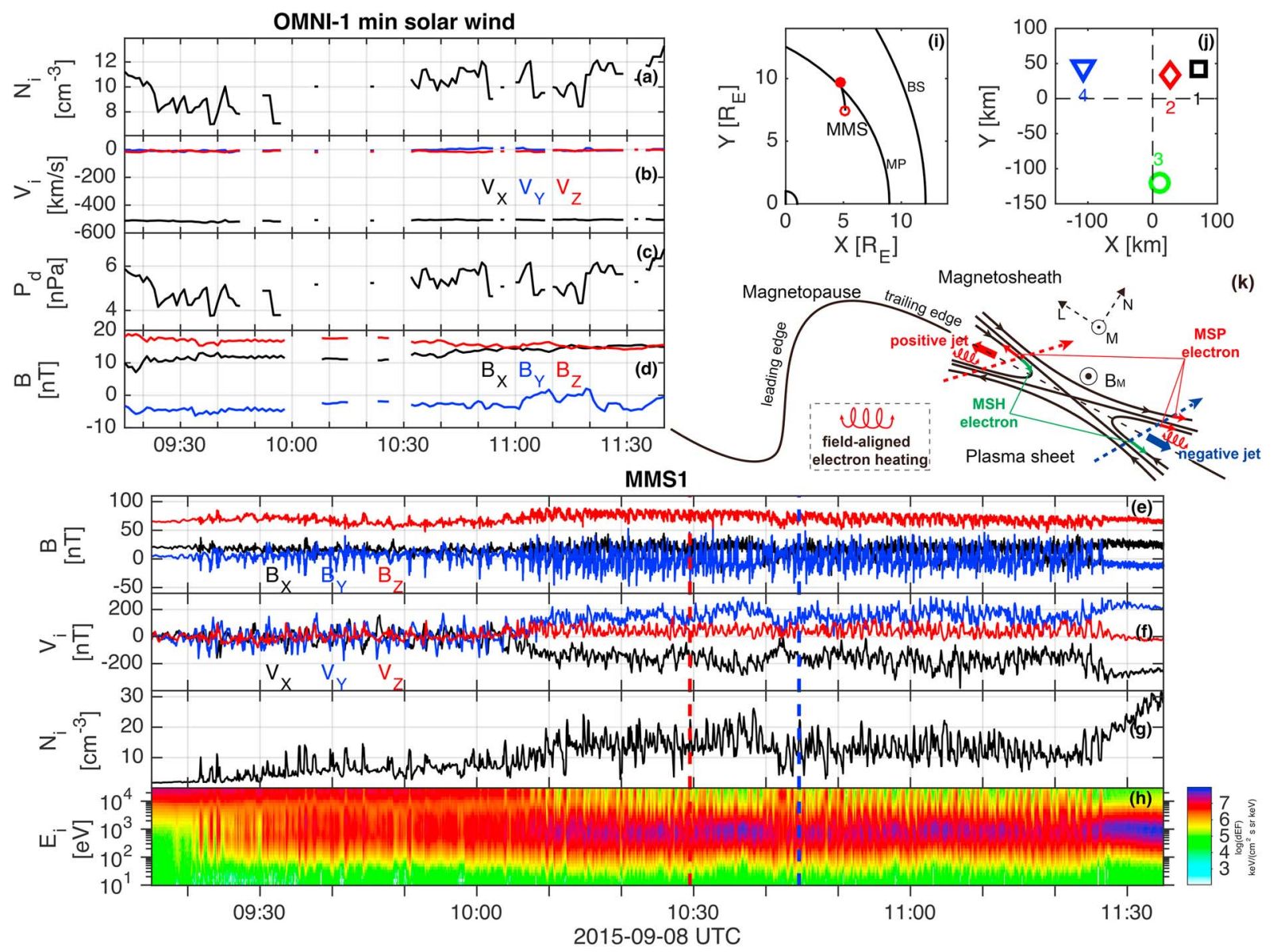

Figure 1. Solar wind conditions from the OMNI $1 \mathrm{~min}$ resolution database and observation of Kelvin-Helmholtz waves during the outbound trajectory of MMS1 on 8 September 2015. (a) Solar wind number density, (b) ion bulk velocity in GSE coordinates, (c) dynamic pressure, and (d) interplanetary magnetic field. MMS1 data of (e) magnetic field, (f) ion bulk velocity at $4.5 \mathrm{~s}$ time resolution, $(\mathrm{g})$ ion number density, and $(\mathrm{h})$ ion omnidirectional differential energy flux. The two dashed lines in the bottom part highlight two intervals for detailed analysis. (i) Location of the MMS spacecraft in GSE $X-Y$ plane and (j) separation of the four spacecraft. (k) A schematic of one trailing edge of the KH wave with reconnection. The two dashed lines with arrows (red and blue) denote the two MMS magnetopause crossings highlighted in Figures $1 \mathrm{e}-1 \mathrm{~h}$.

During the extended northward IMF period, the four MMS spacecraft traversed the duskside magnetopause from the dayside plasma sheet to the magnetosheath, as shown in the overview of MMS1 (Figures 1e-1h). This is clear from the increase in plasma density and velocity (Figures $1 \mathrm{f} \mathrm{and} 1 \mathrm{~g}$ ), and the ion energy flux, which changes from tens of keV at the beginning of the interval to around $1 \mathrm{keV}$ in the end (Figure $1 \mathrm{~h}$ ). Figure $1 \mathrm{i}$ shows the outbound trajectories of MMS in the GSE $X-Y$ plane from 09:15 to 11:40 UT; the separation of the four spacecraft is about 100-150 km (Figure 1j). The MMS magnetic field data is from the Fluxgate Magnetometer [Russell et al., 2014] at $16 \mathrm{~Hz}$ resolution, and particle data are from the FPI instrument [Pollock et al., 2016] at $4.5 \mathrm{~s}$ resolution in this overview figure. We also use the energetic electron data at $0.3 \mathrm{~s}$ resolution from the Fly's Eye Energetic Particle Spectrometer (FEEPS) instrument [Blake et al., 2015] for the detailed study.

There are no significant fluctuations in the intervals of plasma sheet (09:15-09:20 UT) and magnetosheath (11:27-11:35 UT). The magnetic field is persistently northward with $\sim 13^{\circ}$ magnetic shear between the steady plasma sheet and magnetosheath. Figure $1 \mathrm{f}$ shows that magnetosheath ions stream tailward $\left(V_{X}=-260 \mathrm{~km} / \mathrm{s}\right)$ and duskward $\left(V_{Y}=220 \mathrm{~km} / \mathrm{s}\right)$ along the magnetopause, whereas the plasma sheet ions are almost stagnant. During the interval 09:20-11:25 UT, MMS1 observed quasi-periodic perturbations of the plasma and magnetic field parameters during the boundary layer crossing. The boundary layer can be separated into an inner boundary layer (09:20-10:05 UT) with overall stagnant ion flow and an outer boundary layer (10:05-11:25 UT) with magnetosheath-like flow. Since the boundary conditions are steady, these perturbations should be due to local instabilities. We identify these perturbations as surface waves excited by the $\mathrm{KH}$ instability due to the large velocity shear and small magnetic shear, and Eriksson et al. [2016] 
identified the KH instability in detail using the linear criterion [Chandrasekhar, 1961]. The dominant period of the $\mathrm{KH}$ waves is about $68 \mathrm{~s}$ based on fast Fourier transform (FFT) analysis of the density and magnetic field data [Paschmann and Daly, 1998]. The phase velocity of the waves $[-220,186,-11] \mathrm{km} / \mathrm{s}$ is estimated from $90 \%$ of the center-of-mass velocity in the spacecraft rest frame, computed from parameters on both sides of the boundary [Hasegawa et al., 2009]. The wavelength along the magnetopause is $3 R_{E}$ (Earth radius), which is comparable to results from previous observations and simulations [Hasegawa et al., 2006; Li et al., 2012; Lin et al., 2014].

We examine each crossing of the boundary layer for evidence of magnetic reconnection. The dashed lines in Figures $1 \mathrm{e}-1 \mathrm{~h}$ mark two events with one positive (red) and one negative (blue) jet at the magnetopause. The schematic in Figure $1 \mathrm{k}$ shows spacecraft crossings of both the positive and negative jets, with the magnetic field configuration and kinetic signatures of ongoing reconnection. A detailed study of these two events is presented in the next section.

\section{Reconnection Due to KH Waves}

We use the high-resolution ion ( $0.15 \mathrm{~s}$ ) and electron ( $0.03 \mathrm{~s}$ ) data from the FPI instrument for detailed analysis of reconnection due to $\mathrm{KH}$ waves. The particle energy flux and pitch angle distributions are computed directly from the three-dimensional particle distribution function. In this section, two events are investigated, one on either side of the reconnection $\mathrm{X}$ line at the $\mathrm{KH}$ waves active magnetopause.

Figure 2 shows MMS1 time series of a $\mathrm{KH}$ wave trailing edge crossing from the inner boundary layer to the magnetosheath in the LMN frame during the interval 10:29:26-10:29:33 UT (red dashed line in Figures 1e-1h). The inner boundary layer is characterized by low number density $\left(<10 \mathrm{~cm}^{-3}\right.$, Figure $\left.1 \mathrm{c}\right)$ and several keV energy electrons (Figure 1e), while the denser $\left(\sim 20 \mathrm{~cm}^{-3}\right)$ magnetosheath includes colder electrons. The timing analysis of the magnetic field data [Paschmann and Daly, 1998] between 10:29:25 and 10:29:35 UT leads to the direction normal to the boundary $\mathbf{N}=[0.95,0.24,-0.22]$ in GSE, and the boundary normal velocity $\mathbf{V}_{N}=-110 \mathrm{~km} / \mathrm{s}$. The negative sign of $\mathbf{V}_{N}$ means tailward motion of the boundary, which is consistent with the outbound traversal of MMS.

A preliminary $\mathbf{L}$ is the average maximum variance direction over four spacecraft, $u$ sing the minimum variance analysis (MVA) of the magnetic field data from each spacecraft [Paschmann and Daly, 1998]. The $\mathbf{M}$ direction is defined as the cross product of $\mathbf{N}$ (from the timing method) and $\mathbf{L}$, and the final $\mathbf{L}$ completes the right-handed orthogonal system. Here $\mathbf{N}$ from timing analysis is close to the minimum variance directions from all four spacecraft's MVA results. The $\mathbf{L}$ and $\mathbf{M}$ directions for the magnetopause event in Figure 2 are $\mathbf{L}=[-0.25,0.97,-0.02]$ and $\mathbf{M}=[0.21,0.07,0.98]$ in GSE. $\mathbf{L}, \mathbf{M}$, and $\mathbf{N}$ are closely aligned with the GSE $\mathbf{Y}, \mathbf{Z}$, and $\mathbf{X}$ directions, respectively.

The local configuration of the magnetic field can change during the evolution and propagation of $\mathrm{KH}$ waves [Takagi et al., 2006; Hasegawa et al., 2009]. In Figure 2a, the $\mathbf{B}_{L}$ component varies from positive to negative when MMS crosses a density boundary. The local magnetic shear $\sim 51^{\circ}$ is larger than the average shear $\left(\sim 13^{\circ}\right)$, and most of the other trailing edge crossings have a similar magnetic configuration as shown here. The ion flow increases in the $\mathbf{L}$ direction during the boundary interval 10:29:28.45-10:29:30.20 UT, marked by a yellow bar in Figure 2. The width of the boundary $170 \mathrm{~km}$ is about three ion-inertial lengths ( $\sim 50 \mathrm{~km})$ of the magnetosheath side, smaller than average width of the dayside magnetopause [Berchem and Russell, 1982]. The maximum $\mathbf{V}_{L}$ is about $350 \mathrm{~km} / \mathrm{s}$, about $150 \mathrm{~km} / \mathrm{s}$ faster than the adjacent magnetosheath flow.

We perform the Walén test to check if the enhanced flow is a reconnection jet [Sonnerup et al., 1981]. The tangential stress balance test for a rotational discontinuity is achieved by comparing the two vectors $\triangle \mathbf{V}=\mathbf{V}_{j}-\mathbf{V}_{\mathrm{MSH}}$, and $\triangle \mathbf{V}_{A}=\mathbf{V}_{A, j}-\mathbf{V}_{A, \mathrm{MSH}}$. Here $\mathbf{V}_{A}=\sqrt{(1-\alpha) /\left(\mu_{0} \rho\right)} \mathbf{B}$ is the Alfvén velocity, $\mu_{0}$ is the permeability in vacuum, $\rho$ is the mass density of the plasma, $\alpha=\left(P_{11}-P_{\perp}\right) \mu_{0} / B^{2}$ is a pressure anisotropy factor, $P$ is the ion pressure, and MSH and $\mathrm{j}$ denote the magnetosheath and ion jet, respectively. The two vectors are evaluated in the local (L, M) plane tangential to the magnetopause. For the event in Figure 2, the red and blue bars denote the magnetosheath and jet reference regions (Figure 2b), and the result of the Walén test is presented in Table 1. The ratio $R=|\triangle \mathbf{V}| /\left|\triangle \mathbf{V}_{A}\right|$ and the shear angle $\theta$ between these two vectors are also reported for the reconnection jet. The averaged $R=0.96$ and $\theta=32^{\circ}$ from the four spacecraft indicate good agreement with the theoretical predictions for the positive reconnection jet in Figure 2 [Sonnerup et al., 1981]. 


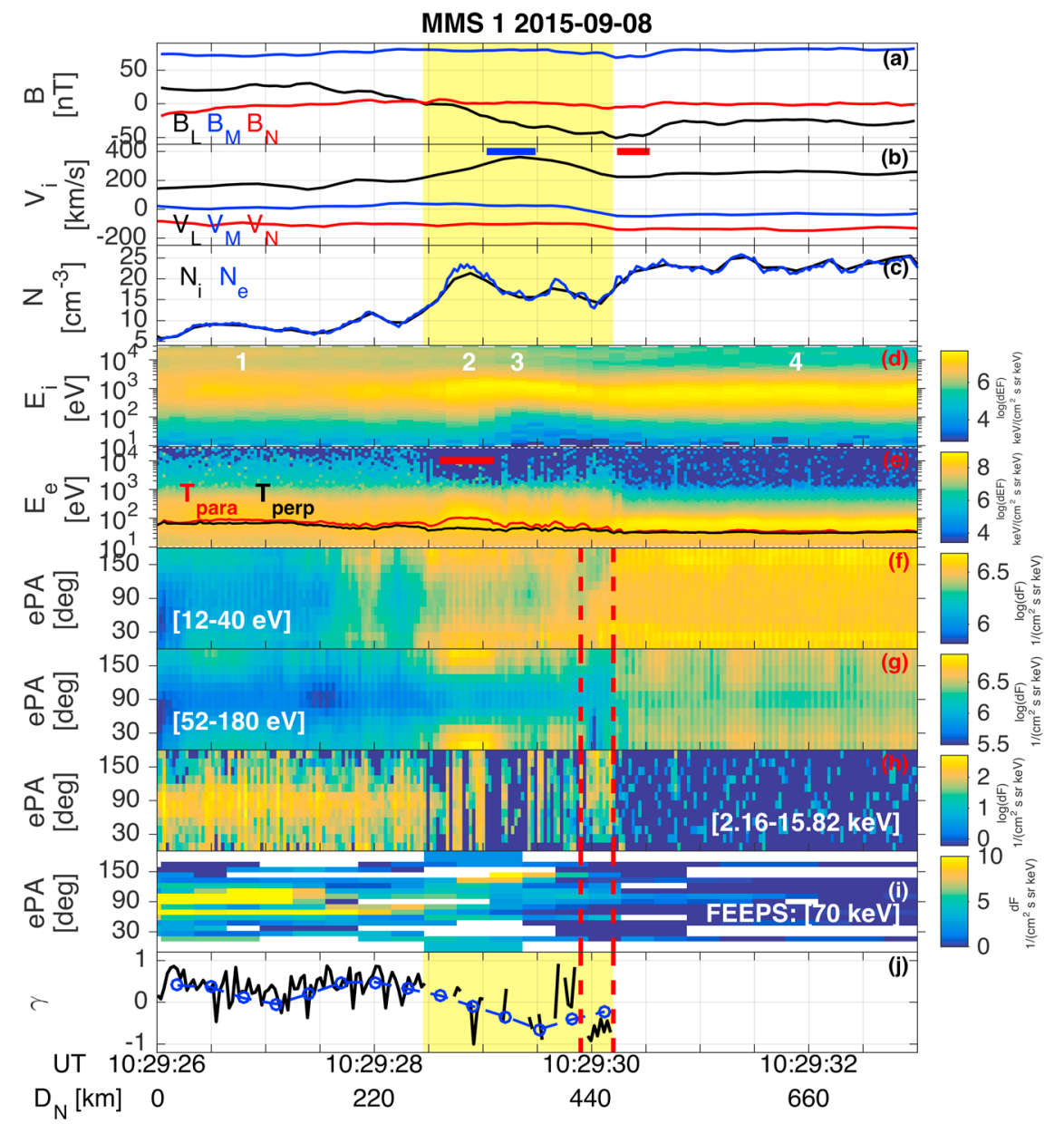

Figure 2. A positive reconnection jet during the $\mathrm{KH}$ wave observation by MMS1. (a) magnetic field in LMN coordinates $(\mathbf{L}=[-0.25,0.97,-0.02], \mathbf{M}=[0.21,0.07,0.98]$, and $\mathbf{N}=[0.95,0.24,-0.22]$ in GSE), (b) ion bulk velocity with marker for jet (thick blue bar) and magnetosheath (thick red bar) used for the Walén test, (c) ion and electron number densities, (d) ion omnidirectional energy flux, (e) electron omnidirectional energy flux with temperature, $(f-i)$ pitch angle distributions of low-energy (12-40 eV), middle-energy (52-180 eV), high-energy (2.16-15.82 keV), and energetic (70 keV) electrons. (j) Asymmetry factor of high-energy and $70 \mathrm{keV}$ electrons. The yellow shaded region indicates a positive jet, and the thick red bar in Figure 2e denotes the region with strong electron temperature anisotropy. The region within the two red dashed lines has strong evidence of inward moving cold magnetosheath electrons and escaping $\mathrm{keV}$ magnetospheric electrons. The four numbers in Figure $2 \mathrm{~d}$ represent the inner boundary layer, the ion density peak in the boundary, the reconnection jet, and the magnetosheath, respectively.

The typical energy of magnetosheath electrons is about $100 \mathrm{eV}$, and we observe a mixture of plasma sheet $(>1 \mathrm{keV})$ and magnetosheath electrons in the inner boundary layer (Figure 2e). On the magnetospheric side of the positive jet 10:29:28.6-10:29:29.1 UT (thick red bar), the electrons have a strong temperature anisotropy $T_{\|} / T_{\perp}$, with a maximum factor of 2.4. Figures $2 \mathrm{f}-2 \mathrm{~h}$ present electron pitch angle distributions for three different energy ranges. The low-energy (12-40 eV) electrons are located mostly in the magnetosheath, and they appear mainly in the field-aligned direction in the boundary. The middle-energy (52-180 eV) electrons occur mostly in the boundary where the strong temperature anisotropy is observed and exhibit clear field-aligned heating, compared with the magnetosheath electron population. The feature of field-aligned electron heating is consistent with ongoing reconnection at the magnetopause [Graham et al., 2014]. The high-energy $(2.16-15.82 \mathrm{keV})$ electrons in the inner boundary layer occur mostly close to $90^{\circ}$ pitch angle.

In Figure $2 \mathrm{~h}$, the interval between two red dashed lines 10:29:29.9-10:29:30.2 UT shows dominance of high-energy $(2.16-15.82 \mathrm{keV})$ electrons closer to the antiparallel over the parallel direction. Figure $2 \mathrm{i}$ shows the pitch angle distributions of the $70 \mathrm{keV}$ energetic electrons, computed from the FEEPS instruments onboard MMS1 and MMS2. The antiparallel flux is higher than the parallel flux around 10:29:29.5 UT. 
Table 1. Results of Walén Test for the Positive and the Negative Jets by Four MMS Spacecraft ${ }^{\mathrm{a}}$

\begin{tabular}{|c|c|c|c|c|c|c|c|c|}
\hline & \multicolumn{5}{|c|}{ Positive Jet } & \multicolumn{3}{|c|}{ Negative Jet } \\
\hline & $\triangle \mathbf{V}(\mathrm{km} / \mathrm{s})$ & $\triangle \mathbf{v}_{A}(\mathrm{~km} / \mathrm{s})$ & $R$ & $\theta$ & $\triangle \mathbf{V}(\mathrm{km} / \mathrm{s})$ & $\triangle \mathbf{v}_{A}(\mathrm{~km} / \mathrm{s})$ & $R$ & $\theta$ \\
\hline MMS1 & {$[128,72]$} & {$[75,94]$} & 1.22 & $22^{\circ}$ & {$[-104,17]$} & {$[243,48]$} & 0.42 & $160^{\circ}$ \\
\hline MMS2 & {$[120,62]$} & {$[64,107]$} & 1.08 & $32^{\circ}$ & {$[-101,12]$} & {$[221,-11]$} & 0.46 & $176^{\circ}$ \\
\hline MMS3 & {$[137,56]$} & {$[85,134]$} & 0.94 & $35^{\circ}$ & {$[-134,9]$} & {$[239,14]$} & 0.56 & $173^{\circ}$ \\
\hline MMS4 & {$[107,37]$} & {$[97,158]$} & 0.61 & $39^{\circ}$ & {$[-145,5]$} & {$[242,-2]$} & 0.60 & $178^{\circ}$ \\
\hline
\end{tabular}

a The tangential stress balance test for a rotational discontinuity is achieved by comparing the two vectors $\left(\triangle \mathbf{V}=\mathbf{V}_{j}-\right.$ $\mathbf{V}_{\mathrm{MSH}}$, and $\left.\triangle \mathbf{V}_{A}=\mathbf{V}_{A, j}-\mathbf{V}_{A, \mathrm{MSH}}\right)$ in the local $(\mathbf{L}, \mathbf{M})$ plane tangential to the magnetopause. Here $\mathbf{V}_{A}=\sqrt{(1-\alpha) /\left(\mu_{0} \rho\right)} \mathbf{B}$ is the Alfvén velocity, $\mu_{0}$ is the permeability in vacuum, $\rho$ is the ion mass density of the plasma, $\alpha=\left(P_{\| 1}-P_{\perp}\right) \mu_{0} / B^{2}$ is a pressure anisotropy factor, $P$ is the ion pressure, and MSH and j denote the magnetosheath and ion jet, respectively. The ratio $R=|\triangle \mathbf{V}| /\left|\triangle \mathbf{v}_{A}\right|$ and the shear angle between these two vectors are also computed. The positive and negative jets are from interval 10:29:26-10:29:33 UT (Figure 2) and 10:44:32-10:44:38 UT (Figure 3), respectively.

We define a factor $\gamma=\left(F_{0^{\circ}-60^{\circ}}-F_{120^{\circ}-180^{\circ}}\right) /\left(F_{0^{\circ}-60^{\circ}}+F_{120^{\circ}-180^{\circ}}\right)$ to quantify this asymmetry in the pitch angle distribution. Here $F_{0^{\circ}-60^{\circ}}$ and $F_{120^{\circ}-180^{\circ}}$ denote the keV electron fluxes in the pitch angle range $0^{\circ}-60^{\circ}$ and $120^{\circ}-180^{\circ}$, respectively. The black curve in Figure $2 \mathrm{j}$ represents the $\gamma$ ratio of the high-energy $(2.16-15.82 \mathrm{keV})$ electrons, while the blue curve with circles is the ratio of the $70 \mathrm{keV}$ energetic electrons. The value of $\gamma(\sim-0.75)$ in the interval 10:29:29.9-10:29:30.2 UT confirms that high-energy electrons occur

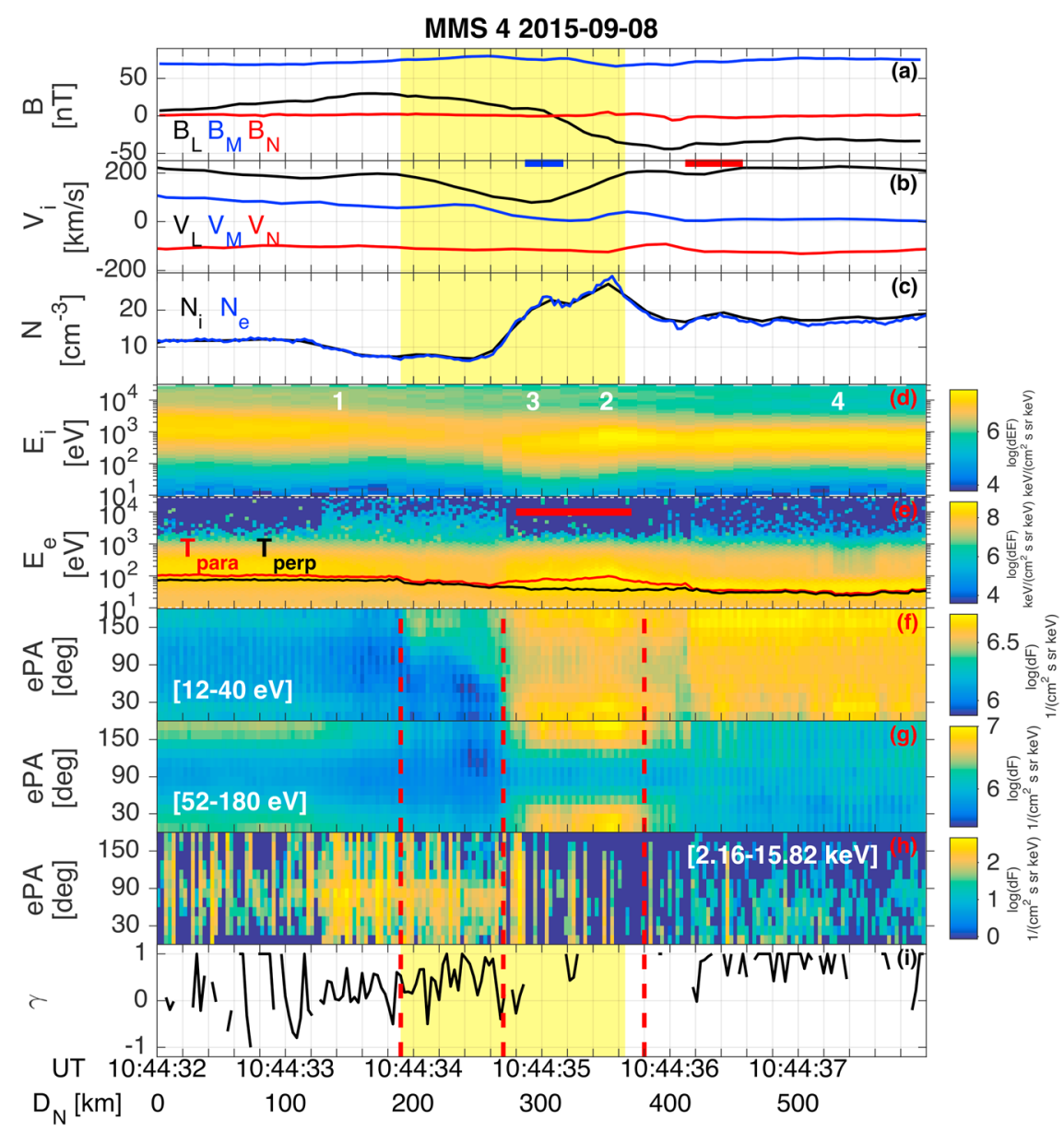

Figure 3. A negative reconnection jet during the KH wave observation by MMS4. The figure is presented in a similar manner as Figure 2, except for the pitch angle distributions of $70 \mathrm{keV}$ energetic electrons. The local LMN coordinates of this magnetopause crossing are $\mathbf{L}=[-0.34,0.93,-0.10], \mathbf{M}=[0.18,0.17,0.97]$, and $\mathbf{N}=[0.92,0.31,-0.23]$ in GSE. (i) The ratio is computed for the electron flux of parallel to antiparallel population. 


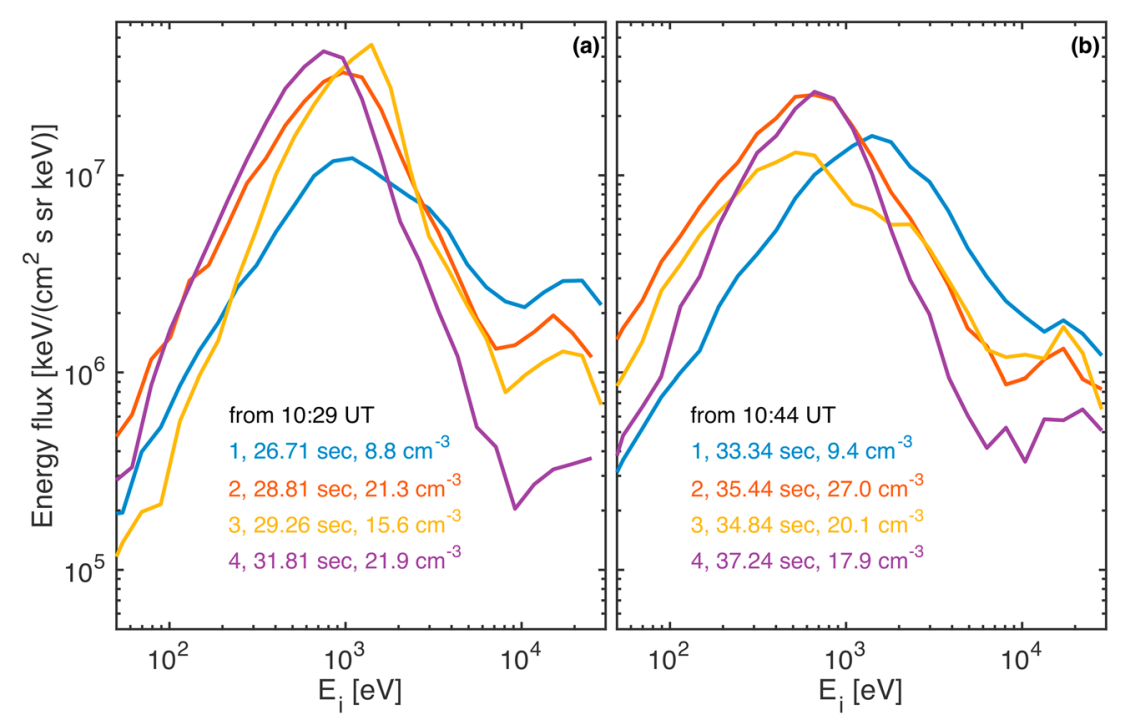

Figure 4. Ion omnidirectional differential energy flux profiles of four points from the (a) positive and (b) negative jets. As shown in Figures $2 d$ and $3 d$, the four points denote the inner boundary layer (1), ion density peak in the boundary (2), reconnection jet (3), and magnetosheath (4), respectively. The inner boundary layer (curve 1 in each panel) is a combination of low-energy magnetosheath and high-energy magnetospheric ions. The low-energy ion population increases gradually during the outbound trajectory, with decreasing high-energy densities. Their signatures are consistent with ion transfer and mixing during ongoing reconnection.

dominantly along the antiparallel direction. The energetic electrons show similar antiparallel pitch angle distributions around 10:29:29.5 UT in the boundary. Also, the highlighted interval coincides with a low-energy electron beam occurring dominantly along the parallel direction (Figure 2f). A schematic of the positive jet crossing is shown in Figure $1 \mathrm{k}$. The directions of low-energy and keV electron beams demonstrate that the magnetosheath electrons move toward the $X$ line along the reconnected magnetic field lines, and the magnetospheric electrons escape into the magnetosheath [Khotyaintsev et al., 2006].

In a similar manner as Figure 2, Figure 3 presents an example of a negative reconnection jet observed by MMS4 (blue dashed line in Figure 1) at another KH wave trailing edge crossing 10:44:32-10:44:38 UT. The $70 \mathrm{keV}$ energetic electron data is not presented in Figure 3 because of the lower counts during this event. The LMN coordinate system determined with timing analysis and the MVA method is $\mathbf{L}=[-0.34,0.93,-0.10], \mathbf{M}=[0.18$, $0.17,0.97]$, and $\mathbf{N}=[0.92,0.31,-0.23]$, still close to the GSE $Y, Z, X$ directions, respectively, as in the previous case. The boundary normal velocity is about $-100 \mathrm{~km} / \mathrm{s}$, and the local magnetic shear angle is about $56^{\circ}$. The interval 10:44:32-10:44:33.2 UT is the outer boundary layer, characterized by intermediate plasma densities between the inner boundary layer and the magnetosheath and a lack of high-energy electrons (Figures $2 \mathrm{e}$ and 2h) [Sckopke et al., 1981]. The yellow shaded region indicates an interval of decreased ion velocity, with a scale of three ion-inertial lengths. The Walén test in Table 1 shows that the decreased flow (thick blue bar) is an Alfvénic negative jet, with a smaller $R$ than the positive jet. The sunward interval 10:44:34.8-10:44:35.7 UT (thick red bar in Figure 2e) of the negative jet exhibits strong electron temperature anisotropy $\left(T_{\| 1} / T_{\perp}\right)$ with a maximum factor 2.8, due to the field-aligned heating (Figure $2 \mathrm{~g}$ ).

The low-energy electrons have a pitch angle distribution closer to the antiparallel direction in the interval (10:44:33.9-10:44:34.7 UT, Figure 2f) within the first two red dashed lines. In Figures $2 \mathrm{~h}$ and $2 \mathrm{i}$, the pitch angle distributions and the factor $\gamma$ show that the high-energy $(2.16-15.82 \mathrm{keV})$ electrons are dominant in parallel direction in the outer edge of the boundary and magnetosheath. The pitch angle feature of the negative jet further confirms that the magnetosheath electrons cross the magnetopause along the reconnected magnetic field lines, and the magnetospheric electrons escape into the magnetosheath (as illustrated in Figure $1 \mathrm{k}$ ).

Magnetosheath plasma is found in the inner boundary layer near the interval of positive and negative jets shown in Figures 2 and 3. We select four points around the jets to illustrate the ion transfer and mixing: 1 for the inner boundary layer, 2 for the density peak in the boundary, 3 for the reconnection jet, and 4 the for magnetosheath (indicated in Figures $2 \mathrm{~d}$ and $3 \mathrm{~d}$ ). The ion omnidirectional differential energy fluxes at these points are presented in Figure 4. The ion distribution (curve 1) of the inner boundary layer combines high-energy 
(>10 keV) magnetospheric ions and low-energy (100 eV-5 keV) magnetosheath ions originating from solar wind for both events. The density of high-energy ions decreases gradually along the outbound trajectory, with increasing low-energy magnetosheath population. Curve 3 in Figure 4a shows the accelerated ion distribution of the positive jet, compared with the magnetosheath ion distribution (curve 4). The negative jet ion has a broader distribution of low-energy ions, with a main peak around $500 \mathrm{eV}$ (curve 3 in Figure 4b). For the positive jet, the maximum ion density $\left(21.3 \mathrm{~cm}^{-3}\right)$ in the boundary reaches the density level of the magnetosheath. For the negative jet, the maximum density is about $50 \%$ larger than the adjacent magnetosheath. For these two jets, the density maximum is on the magnetospheric side for the positive jet and on the magnetosheath side for the negative jet.

\section{Summary and Conclusion}

The high-resolution particle instruments FPI and FEEPS on board the four MMS spacecraft provide the first opportunity to study the kinetic signatures of ongoing reconnection due to $\mathrm{KH}$ waves. We investigate the magnetopause during northward IMF when KH waves are observed by MMS on 8 September 2015, which is associated with reconnection [Eriksson et al., 2016]. In the trailing edges of the $\mathrm{KH}$ wave, the local magnetic shear angle is increased and the magnetopause boundary is as thin as three ion-inertial lengths. We study electron distributions around two Alfvénic ion jets in the trailing edges of $\mathrm{KH}$ waves. One of the jets propagates with the magnetosheath flow and the other against the flow. Both jets show similar kinetic properties. The electron pitch angle distributions show that the magnetosheath electrons cross the magnetopause and the magnetospheric electrons escape into the magnetosheath along the reconnected field lines. The magnetosheath electrons are heated at the high-density edge of the jets, predominantly in the field-aligned direction, which gives a strong temperature anisotropy. The gradual change in ion fluxes at characteristic magnetospheric and magnetosheath energies across the boundary indicates mixing of the two ion populations. We conclude that these observations provide unambiguous kinetic evidence for ongoing reconnection due to $\mathrm{KH}$ waves.

Acknowledgments

The OMNI data were generated by J. H. King and N. Papitashivilli and provided via http://cdaweb.gsfc. nasa.gov/. This work was supported by Swedish National Space Board (SNSB) contracts $164 / 14$ and $176 / 15$. The work at IRAP was supported by CNES and CNRS. MMS observations are publically available via NASA resources and the Science Data Center at CU/LASP (https://lasp.colorado.edu/mms/sdc/ public/). W. Li thanks S. Eriksson and his colleagues as members in MMS scientist-in-the-loop (SITL) team for selecting the 8 September 2015 event.

\section{References}

Berchem, J., and C. T. Russell (1982), The thickness of the magnetopause current layer: ISEE 1 and 2 observations, J. Geophys., 87(A4), 2108-2114, doi:10.1029/JA087iA04p02108.

Blake, J. B., et al. (2015), The fly's eye energetic particle spectrometer (FEEPS) sensors for the magnetospheric multiscale (MMS) mission, Space Sci. Rev., 199, 309-329, doi:10.1007/s11214-015-0163-x.

Burch, J. L., T. E. Moore, R. B. Torbert, and B. L. Giles (2015), Magnetospheric multiscale overview and science objectives, Space Sci. Rev., 199, 5-21, doi:10.1007/s11214-015-0164-9.

Burch, J. L., et al. (2016), Electron-scale measurements of magnetic reconnection in space, Science, doi:10.1126/science.aaf2939, in press. Chandrasekhar, S. (1961), Hydrodynamic and Hydromagnetic Stability, Oxford Univ. Press, New York.

Chaston, C. C., M. Wilber, F. S. Mozer, M. Fujimoto, M. L. Goldstein, M. Acuna, H. Reme, and A. Fazakerley (2007), Mode conversion and anomalous transport in Kelvin-Helmholtz vortices and kinetic Alfvén waves at the Earth's magnetopause, Phys. Rev. Lett., 99, 175004, doi:10.1103/PhysRevLett.99.175004.

Eriksson, S., et al. (2016), Magnetospheric multiscale observations of magnetic reconnection associated with Kelvin-Helmholtz waves, Geophys. Res. Lett., 43, doi:10.1002/2016GL068783.

Faganello, M., F. Califano, and F. Pegoraro (2008), Competing mechanisms of plasma transport in inhomogeneous configurations with velocity shear: The solar-wind interaction with Earth's magnetosphere, Phys. Rev. Lett., 100, 015001, doi:10.1103/PhysRevLett.100.015001.

Fuselier, S. A., W. S. Lewis, C. Schiff, R. Ergun, J. L. Burch, S. M. Petrinec, and K. J. Trattner (2014), Magnetospheric multiscale science mission profile and operations, Space Sci. Rev., 199, 77-103, doi:10.1007/s11214-014-0087-x.

Gosling, J. T., M. F. Thomsen, S. J. Bame, T. G. Onsager, and C. T. Russell (1990), The electron edge of low latitude boundary layer during accelerated flow events, Geophys. Res. Lett., 17(11), 1833-1836, doi:10.1029/GL017i011p01833.

Graham, D. B., Y. V. Khotyaintsev, A. Vaivads, M. André, and A. N. Fazakerley (2014), Electron dynamics in the diffusion region of an asymmetric magnetic reconnection, Phys. Rev. Lett., 112, 215004, doi:10.1103/PhysRevLett.112.215004.

Hasegawa, H., M. Fujimoto, T. D. Phan, H. Rème, A. Balogh, M. W. Dunlop, C. Hashimoto, and R. TanDokoro (2004), Transport of solar wind into Earth's magnetosphere through rolled-up Kelvin-Helmholtz vortices, Nature, 430(7001), 755-758, doi:10.1038/nature02799.

Hasegawa, H., M. Fujimoto, K. Takagi, Y. Saito, T. Mukai, and H. Rème (2006), Single-spacecraft detection of rolled-up Kelvin-Helmholtz vortices at the flank magnetopause, J. Geophys. Res., 111, A09203, doi:10.1029/2006JA011728.

Hasegawa, H., et al. (2009), Kelvin-Helmholtz waves at the Earth's magnetopause: Multiscale development and associated reconnection, J. Geophys. Res., 114, A12207, doi:10.1029/2009JA014042.

Henri, P., F. Califano, M. Faganello, and F. Pegoraro (2012), Magnetised Kelvin-Helmholtz instability in the intermediate regime between subsonic and supersonic regimes, Phys. Plasmas, 19(7), 072908, doi:10.1063/1.4739234.

Karimabadi, H., et al. (2013), Coherent structures, intermittent turbulence, and dissipation in high-temperature plasmas, Phys. Plasmas, 20(1), 012303, doi:10.1063/1.4773205.

Khotyaintsev, Y. V., A. Vaivads, A. Retinò, M. André, C. J. Owen, and H. Nilsson (2006), Formation of inner structure of a reconnection separatrix region, Phys. Rev. Lett., 97, 205003, doi:10.1103/PhysRevLett.97.205003.

Knoll, D. A., and L. Chacón (2002), Magnetic reconnection in the two-dimensional Kelvin-Helmholtz instability, Phys. Rev. Lett., 88, 215003, doi:10.1103/PhysRevLett.88.215003. 
Li, W., C. Wang, B. Tang, X. Guo, and D. Lin (2013), Global features of Kelvin-Helmholtz waves at the magnetopause for northward interplanetary magnetic field, J. Geophys. Res. Space Physics, 118, 5118-5126, doi:10.1002/jgra.50498.

Li, W. Y., X. C. Guo, and C. Wang (2012), Spatial distribution of Kelvin-Helmholtz instability at low-latitude boundary layer under different solar wind speed conditions, J. Geophys. Res., 117, A08230, doi:10.1029/2012JA017780.

Lin, D., C. Wang, W. Li, B. Tang, X. Guo, and Z. Peng (2014), Properties of Kelvin-Helmholtz waves at the magnetopause under northward interplanetary magnetic field: Statistical study, J. Geophys. Res. Space Physics, 119, 7485-7494, doi:10.1002/2014JA020379.

Ma, X., A. Otto, and P. A. Delamere (2014), Interaction of magnetic reconnection and Kelvin-Helmholtz modes for large magnetic shear: 1. Kelvin-Helmholtz trigger, J. Geophys. Res. Space Physics, 119, 781-797, doi:10.1002/2013JA019224.

Mitchell, D. G., F. Kutchko, D. J. Williams, T. E. Eastman, L. A. Frank, and C. T. Russell (1987), An extended study of the low-latitude boundary layer on the dawn and dusk flanks of the magnetosphere, J. Geophys. Res., 92(A7), 7394-7404, doi:10.1029/JA092iA07p07394.

Miura, A., and P. L. Pritchett (1982), Nonlocal stability analysis of the MHD Kelvin-Helmholtz instability in a compressible plasma, J. Geophys. Res., 87(A9), 7431-7444, doi:10.1029/JA087iA09p07431.

Nakamura, T. K. M., and W. Daughton (2014), Turbulent plasma transport across the Earth's low-latitude boundary layer, Geophys. Res. Lett., 41, 8704-8712, doi:10.1002/2014GL061952.

Nakamura, T. K. M., and M. Fujimoto (2006), Magnetic reconnection within MHD-scale Kelvin-Helmholtz vortices triggered by electron inertial effects, Adv. Space Res., 37(3), 522-526, doi:10.1016/j.asr.2005.01.057.

Nakamura, T. K. M., M. Fujimoto, and A. Otto (2008), Structure of an MHD-scale Kelvin-Helmholtz vortex: Two-dimensional two-fluid simulations including finite electron inertial effects, J. Geophys. Res., 113, A09204, doi:10.1029/2007JA012803.

Nakamura, T. K. M., W. Daughton, H. Karimabadi, and S. Eriksson (2013), Three-dimensional dynamics of vortex-induced reconnection and comparison with THEMIS observations, J. Geophys. Res. Space Physics, 118, 5742-5757, doi:10.1002/jgra.50547.

Nykyri, K., and A. Otto (2001), Plasma transport at the magnetospheric boundary due to reconnection in Kelvin-Helmholtz vortices, Geophys. Res. Lett., 28(18), 3565-3568, doi:10.1029/2001GL013239.

Paschmann, G., and P. W. Daly (1998), Analysis Methods for Multi-Spacecraft Data, ESA Publ. Div., Noordwijk, Netherlands.

Perreault, P., and S. Akasofu (1978), A study of geomagnetic storms, Geophys. J. Int., 54(3), 547-573.

Pollock, C., et al. (2016), Fast plasma investigation for magnetospheric multiscale, Space Sci. Rev., 199, 331-406, doi:10.1007/s11214-016-0245-4.

Pu, Z. Y., M. Yei, and Z. X. Liu (1990), Generation of vortex-induced tearing mode instability at the magnetopause, J. Geophys. Res., 95(A7), 10,559-10,566, doi:10.1029/JA095iA07p10559.

Russell, C. T., et al. (2014), The magnetospheric multiscale magnetometers, Space Sci. Rev., 199, 189-256, doi:10.1007/s11214-014-0057-3. Sckopke, N., G. Paschmann, G. Haerendel, B. U. Ö. Sonnerup, S. J. Bame, T. G. Forbes, E. W. Hones, and C. T. Russell (1981), Structure of the low-latitude boundary-layer, J. Geophys. Res., 86(A4), 2099-2110, doi:10.1029/JA086iA04p02099.

Song, P., and C. T. Russell (1992), Model of the formation of the low-latitude boundary layer for strongly northward interplanetary magnetic field, J. Geophys. Res., 97(A2), 1411-1420, doi:10.1029/91JA02377.

Sonnerup, B. U. Ö., G. Paschmann, I. Papamastorakis, N. Sckopke, G. Haerendel, S. J. Bame, J. R. Asbridge, J. T. Gosling, and C. T. Russell (1981), Evidence for magnetic field reconnection at the Earth's magnetopause, J. Geophys. Res., 86(A12), 10,049-10,067, doi:10.1029/JA086iA12p10049.

Takagi, K., C. Hashimoto, H. Hasegawa, M. Fujimoto, and R. TanDokoro (2006), Kelvin-Helmholtz instability in a magnetotail flank-like geometry: Three-dimensional MHD simulations, J. Geophys. Res., 111, A08202, doi:10.1029/2006JA011631.

Taylor, M. G. G. T., et al. (2008), The plasma sheet and boundary layers under northward IMF: A multi-point and multi-instrument perspective, Adv. Space Res., 41(10), 1619-1629, doi:10.1016/j.asr.2007.10.013.

Terasawa, T., et al. (1997), Solar wind control of density and temperature in the near-Earth plasma sheet: WIND/GEOTAIL collaboration, Geophys. Res. Lett., 24(8), 935-938, doi:10.1029/96GL04018.

Wing, S., et al. (2014), Review of solar wind entry into and transport within the plasma sheet, Space Sci. Rev., 184(1-4), 33-86, doi:10.1007/s11214-014-0108-9. 